\title{
Effects of experiencing the role of imaginary future generations in decision-making: a case study of participatory deliberation in a Japanese town
}

\author{
Keishiro Hara ${ }^{1,2,3} \cdot$ Yoko Kitakaji $^{4} \cdot$ Hiroaki Sugino $^{5} \cdot$ Ritsuji Yoshioka $^{6} \cdot$ Hiroyuki Takeda $^{1} \cdot$ Yoichi Hizen $^{7,8}$. \\ Tatsuyoshi Saijo ${ }^{3,8,9}$
}

Received: 19 December 2019 / Accepted: 24 January 2021 / Published online: 18 February 2021

(c) The Author(s) 2021

\begin{abstract}
To ensure sustainability, overcoming intergenerational conflict is vital, and social systems supporting decision-making that takes into account the benefits to future generations is thus critically important. One promising approach in such social systems is introducing "imaginary future generations" who act as representatives for the benefits of future generation in actual, present-day decision-making situations. In this study, we explore the effects and implications of participants' experiences as representatives of imaginary future generation. We conducted a citizens' participatory debate on creating a vision and appropriate policies associated with public facilities and housing in a town in Japan, and examined how the thinking patterns and decisions of the participants shifted as a result of debating from the perspectives of both current and imaginary future generations. Based on analyses of a questionnaire and the keywords in answers to a worksheet provided to the participants, we demonstrate that through their experiences as representatives of imaginary future generations, a clear shift in perspective occurred, with increases in self-reflective viewpoint. We also found that the shared viewpoints of the current and future generations existed within the individuals. These findings hint at how we can develop institutions and social systems that facilitate sustainable decision-making.
\end{abstract}

Keywords Imaginary future generations $\cdot$ Future design $\cdot$ Participatory deliberation $\cdot$ Viewpoint sharing $\cdot$ Futurability

Handled by Annamaria Di Fabio, Universita degli Studi di Firenze Dipartimento di Scienze della Formazione e Psicologia (sezione di Psicologia), Italy.

Keishiro Hara

hara@ceids.osaka-u.ac.jp

1 Graduate School of Engineering, Osaka University, Suita, Japan

2 Research Institute of Economy, Trade and Industry, Tokyo, Japan

3 The Tokyo Foundation for Policy Research, Tokyo, Japan

4 Hiroshima University, Higashihiroshima, Japan

5 The University of Tokyo, Tokyo, Japan

6 Yahaba, Iwate, Japan

7 School of Economics and Management, Kochi University of Technology, Kochi, Japan

8 Research Institute for Future Design, Kochi University of Technology, Kochi, Japan

9 Research Institute for Humanity and Nature, Kyoto, Japan

\section{Introduction}

In recent decades, a variety of complex problems, ranging from climate change to ecosystem degradation, have emerged and are now posing serious threats to the sustainability of our societies. For example, anthropogenic Greenhouse gas emissions have been steadily increased over the last decades (IPCC 2014). Rockström et al. (2009) identified nine domains that are essential to the maintenance of the comparatively stable Holocene environment that has been in place for more than 10,000 years and proposed acceptable levels (thresholds) for these planetary boundaries. It has been demonstrated that acceptable levels have already been breached with respect to climate change, rate of biodiversity loss, and the cycles of biochemical substances, such as nitrogen. Such evidence clearly indicates that sustainability presents a massive challenge despite the fact that various promising technologies, policies, and visions have emerged.

Considering these complex sustainability issues, sustainability research has been vigorously pursued over 
recent decades, proposing alternative visions and a synthesis of disciplines (Kates et al. 2001; Clark and Dickson 2003; Komiyama and Takeuchi 2006). Along with these research efforts, a variety of methods using creative activities have been developed and applied in order to expand the capacity of people to envision the future and to detail future policy options via innovative participatory approaches (Iacovidou and Wehrmeyer 2014; Ligtvoet et al. 2016; Eickhoff and Geffer 2009).

One of the interesting questions associated with our search for sustainability solutions centers on how we might actively incorporate the viewpoint of future generations in any vision setting and decision-making so as to reconcile important intergenerational conflicts and tradeoffs. In devising strategies that will ensure sustainability, decisionmaking that takes into account the benefits to future generations are critically important. The essential problem with this approach, however, is that it is difficult to bring the perspective of future generations into present day discussions in any concrete way. As a result, decisions made and measures taken today tend to be biased toward the present without regard to their impact on subsequent generations; thus, leaving the issue of intergenerational conflicts unresolved. We argue that this point is a fundamental issue in the sustainability challenges we face (Saijo 2020a, 2019, 2015; Hara et al. 2019; Sherstyuk et al. 2016).

Saijo (2018) argues that natural human characteristics such as impulse (Sapolsky 2012) and optimism about the future (Sharot 2011), as well as societal systems, such as markets, influence the distribution of resources to satisfy the needs of the present rather than those of the future. These factors lead to shortsighted decision-making that is often at odds with the best interests of future generations. It appears to be fundamentally alien to the thinking of the current generation to behave and make decisions in such a way as to consider their impact on some abstract conception of a future generation. Although other-regarding preferences are also part of human nature (Fischbacher et al. 2001; Fehr-Duda and Fehr 2016), it is immensely difficult for individuals in the present to continually make decisions that will—eventually—benefit future generations at the expense of their own (Hara et al. 2019).

According to Saijo (2019), a person exhibits futurability when he or she experiences an increase in happiness as a result of deciding and acting to forego current gains in order to enrich future generations, and the design and praxis of a society generating futurability is called Future Design (see also Saijo $(2018,2020 b)$ ). We argue that activating futurability through the design of appropriate social systems is crucial to achieving the sustainability of society, overcoming shortsighted decision-making and resolving intergenerational conflicts.
One promising approach that has been shown to activate futurability is creating an "imaginary future generation" as a stakeholder tasked with representing his/her future generation in negotiations with present-generation decisionmaking groups (Saijo 2020b, 2019, 2017, 2015; Kamijo et al. 2017; Hara and Saijo 2017; Hara et al. 2019; Uwasu et al. 2020). Previous research has demonstrated that incorporating imaginary future generations into discussions of issues that will have future impact is an effective way to overcome shortsighted decision-making in certain groups. In the first lab-scale experiment, groups that included an imaginary future generation demonstrated the capacity to make judgments and decisions that opted to leave resources for future generations, even if that meant reducing the remuneration that the group itself would realize (Kamijo et al. 2017). The beneficial effects of creating an imaginary future generation in visioning deliberations and decision-making practices involving citizen participation have also been demonstrated (Hara et al. 2019; Hara and Saijo 2017). For example, adopting a process in which current and imaginary future generation groups form a pair and negotiate to reach an intergenerational consensus in formulating policy or making decisions has been shown to be an effective means to facilitate intergenerational consensus building and to produce proposals that benefit both current and future generations. In the same setting, it was also observed that there was a stark contrast in priority and normativity regarding future society between the current and imaginary future generation groups. For instance, measures proposed by the imaginary future generation group were primarily characterized as utilizing existing local resources, while the present generation groups aimed more at solving current problems, creating conflicts between the current and future generations groups especially at the beginning of negotiation (Hara et al. 2019).

Furthermore, based on the intensive interviews with participants who experienced this type of approach, it was demonstrated that the experience can lead to the creation of a higher level of overarching perspectives of both current and future generations within the individual and that the effects of the process are sustained for some time (robustness). Moreover, those who experienced the imaginary future generations felt intellectually superior (Nakagawa and Saijo 2020; Nakagawa et al. 2017; Hara et al. 2019). These observations tend to support our hypothesis regarding the activation of "futurability".

Although practices involving the inclusion of imaginary future generation groups as stakeholders and active debate partners in decisions being made in the present have shown some positive effects in terms of managing and resolving intergenerational conflicts (Hara et al. 2019), challenges regarding the practical application of this approach remain. Consensus building among current and future groups is likely to require intensive negotiation, which means the 
number of debates and the number of negotiating sessions may be quite large. Further, how the judgement and ways of thinking of participants in such an arrangement change and evolve has not been sufficiently investigated.

Using the intergenerational sustainability dilemma game (abbreviated as ISDG; Kamijo et al. 2017), Shahrier et al. (2017) conducted an experiment in which the participants experienced the standpoints of both the present and next generations in their deliberations. The approach turned out to be effective in detaching participants from their self-interests and inducing them to consider the impact of their decisionmaking on future generations. However, it has not been fully determined whether enabling participants to experience the perspectives of both current and future generations in "realworld" participatory deliberations actually leads to a selfreflective viewpoint that takes into account the interests and inputs of future generations. If allowing debate participants to experience the roles of both current and future generations can be shown to be an effective mechanism for producing such self-reflection, applying the approach to actual policy-making decisions in pursuit of sustainability becomes a highly attractive feature.

In this study, we conducted participatory deliberations among local citizens on the theme of future vision design for public facilities and housing in the town of Yahaba, Iwate Prefecture, Japan. All participants experienced the debate from both the perspective of the current generation and the perspective of an imaginary future generation, successively playing each of the two roles. We then examined how the thinking patterns and judgements of participants changed as a result of their experience. In doing so, we sought to explore the effects of experiencing the role of an imaginary future generation and the prospects of creating a self-reflective process through the activation of futurability.

\section{Case study and methods}

\section{Setting of participatory deliberation}

As noted, a future design debate was conducted in the town of Yahaba in Iwate Prefecture, Japan. Yahaba is a commuter town bordering on the capital city of Morioka, with a population of approximately 27,000 . The town has held public participatory debates repeatedly over many years and is widely known for realizing a water supply vision developed by its citizens. In arranging the debate featured in this study, university researchers (including the authors) and Yahaba local government employees worked with local citizens to ensure a suitable forum. The theme of the debate was designing a vision for the management of public facilities and housing, as described below.
The participatory deliberations were held over three sessions, on January 14, February 12, and March 4, 2017. The debate time was $2.5 \mathrm{~h}$ per session. The citizens participating in the debates were selected by extracting 1,000 names at random from a resident register, mailing invitations to all selected clarifying that participants would be rewarded for their participation, and then choosing the first 26 who responded affirmatively to the invitation. Ages ranged from 20 to 80s. Taking age and gender balance into account, the 26 participants were divided into four groups (A, B, C, D), which remained fixed over the three sessions. On all three occasions, the four groups engaged in debate in separate rooms to remain uninfluenced by the debates of the other groups. At the end of the third session, as a wrapping-up exercise, all four groups gathered together and shared their ideas from the three sessions with all the other participants.

The debate themes were "Propose a vision for public housing in 2050 and relevant policy measures" for groups $\mathrm{A}$ and $\mathrm{B}$, and "Propose a vision for public facilities management in 2050 and relevant policy measures" for groups $\mathrm{C}$ and $\mathrm{D}$. Prior to the debates, we provided the participants with basic information relating to public facilities management and public housing, e.g., locations of facilities in the form of geographic information, initial investment expenses of facilities, expenses incurred in managing and operating facilities and housing, and utilization-related circumstances, to enable the groups to engage in detailed, informed debate.

The structure of the deliberations is described in Fig. 1. For designing deliberation processes, we referred to Shahrier et al. (2017) and Timilsina et al. (2019). In the experiment conducted by Shahrier et al. (2017), participants played the ISDG, in which a sequence of six generations with three members each subsequently chose either an ownpayoff maximizing option or a sustainable option. In the choice, each generation first imagined that they were the next generation members and requested their previous generation regarding which option they wanted their previous generation to choose. They then returned to their original standpoint and chose one of the two options. If their choice matched the request they made from the next generation's standpoint, it became their final choice. Otherwise, the three group members had a vote to determine their final choice.

On the other hand, in the present study, participants first discussed the visions for public facilities management in the future and relevant policy measures from the current generation's perspective. They then did the same as an imaginary future generation. Finally, they introspected between the first and second sessions and proposed relevant policies with the reasons for their proposals and advice to the future generation. Letting participants express the reasons and advice is based on the experiment by Timilsina et al. (2019). In comparison with Shahrier et al. (2017), our experimental design's uniqueness lies in taking the future generation's 
Fig. 1 Structure of the future design deliberations
First session (as the current generation)

All groups think about the future image of Yahaba town in 2050 and then give opinions on the policies and measures for public housing or facilities

Second session (as an imaginary future generation)

All groups think about the image of Yahaba town in 2050 and then give opinions on the policies and measures for public housing or facilities as an "imaginary future generation."

Third session (introspect between the first and second sessions) All groups introspect between the first and second sessions and propose a plan for Yahaba town (a vision for Yahaba town in 2050) together with the reason. They finally provide advice to the future generation who face the decision making on public housing or facilities.

standpoint second and deciding what to propose finally as a group through discussion. Details of our procedures are explained below.

In the first session (January 14), each group engaged in the debate on its theme and on policy measures to address its vision. No particular conditions were imposed on the way to proceed with the debate. Thus, in this first debate, the groups engaged in debate from the viewpoint of the "current generation".

In the second session (February 12), we asked all participants to engage in debate on their 2050 vision as an "imaginary future generation". Our instructions were that "they should identify as citizens of 2050 and debate from that standpoint to advocate for the interests of their generation," and also that "their role is to consider issues not from their own viewpoint as the current generation or that of their families, but rather from that of the future generation". We also tried to help the participants understand the significance of taking part in the debate as representatives of a future generation by explaining specific examples, e.g., climate change. As this suggests, this kind of instruction is an important element in ensuring that the participants maintain their role as the imaginary future generation. Specifically, to encourage and motivate participants to identify as citizens of 2050 (i.e., the imaginary future generation), we asked them to "time-travel" to the year 2050 while remaining at their current age. When speaking as the imaginary future generation, participants were also asked to wear a happi coat, a traditional Japanese coat or jacket often worn during festivals, to remind them that they were representing the future generation. In the first half of the debate, we asked participants to share their images of the state of Yahaba in 2050 and to describe how societal conditions-industrial and socioeconomic conditions, lifestyles, etc.- - appeared to them from the standpoint of their future generation. In the second half of the debate, again from the standpoint of the future generation, we asked participants to envision images for their respective themes (i.e., public facilities/public housing) in 2050 and to discuss policy measures to address them. Thus, the conditions given to the participants were the same between the first and second sessions, except for the difference in the standpoints of the current or imaginary future generations.

In the third session (March 4), we allowed the groups to choose the perspectives for debating, based on their experiences in the first session (debate as the current generation) and the second session (debate as an imaginary future generation). However, we imposed the condition that they should clearly express the reasons for their vision and the policies they proposed and discuss advice to the next generation (Timilsina et al. 2019).

In each session, we sought to promote effective debate by enabling the opinions being offered to be quickly visualized. To this end, we appointed a town hall facilitator and a writer who would record on paper the spoken words of the participating members. To ensure that all groups debated under the same conditions, we unified the content and timing of the facilitators' remarks across the four groups.

At the end of each of the three sessions, the participants were asked to fill out a questionnaire and to complete a worksheet (as will be explained below). The data obtained from these two instruments were used for analyses. 


\section{Analysis methods of data}

\section{Questionnaire survey}

At the end of each session, participants were asked to fill out a follow-up questionnaire that was distributed and collected by the group's facilitator. On their questionnaire, participants were instructed to write a unique personal ID that only they would know. Each ID was the sum of two 4-digit numbers, one corresponding to the participant's year of birth and the other corresponding to the participant's month and day of birth. This ID was used across the three debate sessions on all submitted sheets so that the responses of each individual in each session could be aligned.

The questionnaires included a combination of indicators to evaluate the basic hypothesis of the study-that experience in thinking as a member of both current and imaginary future generations will affect the perceptions and policy formulation processes of individuals when it comes to issues of sustainability. Specifically, the aim of the questionnaire was to shed light on the impact that debating from the standpoint of an imaginary future generation would have on the perceptions of participants and to examine what kinds of policies would be considered desirable by individuals assigned to assume the perspective of both current and future generations in the design and management of public facilities and housing.

We hypothesized that the effects of thinking as a member of an imaginary future generation on participants in the study could be broadly divided into three categories: (1) a change in their perception of the relationship between current and future generations, (2) a change in their evaluation of Yahaba as a town, and (3) their perception of the important aspects of policy-making. Items relating to these three areas were included in the questionnaire (see Online Appendix 1).

The relationship between current and future generations is, on the one hand, oppositional, in that decisions made by the current generation may well deprive the future generation of important resources. On the other hand, it can also be considered a relationship of supporter and supported to the extent that the current generation passes resources on to the future generation by sacrificing its own interests. Latané and Darley (1970) proposed a five-step model for decision-making that leads to helping behavior. The five steps comprise the following: (1) awareness of the event, (2) understanding the urgency of the problem, (3) perceiving responsibility, (4) choosing an appropriate method of helping, and (5) implementing the intervention. Although each of these steps is important, the key step appears to be perceiving responsibility (Latané and Darley 1970). In situations, where the number of potential helpers is very large, a weakening diffusion of responsibility may occur. Moreover, members of the current generation may choose to blame previous generations for consuming future resources. Accepting that the current generation is responsible for problems related to the future generation can lead to helping behavior that serves to protect the interests of future generations.

Sixteen items relating to the relationship between current and future generations were included in the session questionnaires; for example, "The people of today are responsible for the issues being debated," "We must pass on to future generations the things we in the present are enjoying," and "The themes discussed in this debate are serious problems now". Nine items were related to participant evaluations of Yahaba. These included such statements as "Yahaba will probably be a comfortable place to live in 2050" and "Finances/population/welfare is a serious problem for Yahaba". Responses to both sets of items were scored on a 5-point scale, from "1: Totally disagree" to " 5 : Very much agree". Participant perceptions of what policy-making aspects were most important were the focus of eight items, including "Those policies are feasible," and "Leaving room for future people to make their own decisions". Responses to these items were scored on a 5-point Likert scale (1: Totally unimportant; 5: Very important).

To assess participant perspectives on taking the point of view of the current generation versus the point of view of the future generation, we included two items: "In today's debate, I thought about things from the standpoint of people living now," and "In today's debate, I thought about things from the standpoint of future generations" in the survey following the third session only, after the participants had played both their current generation (first session) and future generation (second session) roles. These items were also scored on a 5-point scale, ranging from " 1 : Totally disagree" to " 5 : Very much agree".

Twenty-six participants responded to the questionnaire in the first session ( 8 males, 18 females); 24 responded in the second session ( 8 males, 16 females), and 20 responded in the third session ( 7 males, 13 females). Not all participants were able to participate in the second and third sessions due to scheduling conflicts or for other reasons.

The data were statistically analyzed using SAS. A repeated measures one-way analysis of variance (ANOVA) was conducted on the questionnaire responses from sessions 1, 2 and 3, followed by Tukey's test.

\section{Worksheets}

At the end of each session, participants were also asked to fill out worksheets on which they described the essential points of the policy measures and ideas that they had proposed during the group debates. Since participants were assigned to tackle only one of the two themes (the maintenance and management of public facilities or public 
housing), worksheets relevant to the appropriate theme were distributed to the proper groups. Aside from this, the contents of the worksheets were identical in every respect.

The worksheet items were divided into four main sections: (1) concepts and viewpoints proposed in debating, (2) overall principles and policies, (3) individual principles and policies, and (4) time scale. Table 1 shows the items in detail. Since the main focus of the study was to examine whether the experience of participants in thinking from the perspectives of current and imaginary future generations through the three debate sessions gave rise to a self-reflective viewpoint and changes in thinking patterns, we specifically examined keywords written by participants under the category of (1) concepts and viewpoints proposed in debating. We assumed that these would best provide essential points regarding the ways of thinking and the ideas of individuals.

In analyzing the worksheets, we focused on how the descriptions of the participants changed over the three sessions. We used a text mining (TM) technique capable of breaking down text strings as target data. The flow of analysis was as follows: (1) chunk-down the sentences into morphemes (minimal meaningful language units); (2) create a co-occurrence network diagram for each session; (3) conduct a semantic analysis of each description and digitize it (vectorization); and (4) compare all three sessions. These analyses were applied to item (a) concepts and vision and item (b) important viewpoints when deciding concepts in (1) concepts and viewpoints proposed in debating. However, when digitizing the descriptive responses to (b), we weighted the responses according to the degree of importance.

TM is a quantitative analysis technique that allows objectivity to be retained and arbitrariness to be excluded. It has evolved rapidly in recent years with improvements in computer processing power and the development and the widespread dissemination of software for the analysis. Many studies using TM have been performed in fields ranging from psychology and medicine to marketing. In such studies, quantitative and qualitative methods are not regarded as separate and exclusive, but rather as degrees on a continuum (Lazarsfeld and Barton 1951), and the recursive use of both methods is recommended. In this study, as well, after conducting our quantitative analysis, we reviewed the data with the aim of examining it more deeply.

We mainly used the open software R Ver. 3.2.3 for our statistical analysis, as well as the TM-capable package $\mathrm{RMeCab}$ and the network analysis package "igraph". In accordance with Fries (1952), we narrowed our analysis to the nouns, verbs, and adjectives in the description text, as these are the words that carry semantic content. In creating co-occurrence networks, in accordance with the technique detailed by Sugino et al. (2017), we first expressed the degree of co-occurrence of the most frequent words extracted by morphological analysis (dividing a text into the individual words of the smallest word units that carry meaning) as an adjacency matrix. Then, using a co-occurrence network that was computed via graph theory based on this matrix and grouping the extracted words, we were able to visualize the clusters of co-occurrences of frequent words. For the detection of the clusters (called "communities," which can be interpreted as topics in the context of semantic analysis), we used the modularity $(Q)$ value (Clauset et al. 2004), employing the greedy algorithm featured in a previous study (Fortunato 2010). After creating the co-occurrence network, we digitized the reference density of each community obtained from the network based on the product of the text of respondents and the word frequency matrix, as implemented by Suga et al. (1993). We then applied a multiple factor analysis and visualized the mutual correlation with the intensity of each topic (community) as a vector on the correlation circle. To analyze the degree of importance, we applied a two-dimensional cluster analysis to data weighted by the degree of importance (No. $1 \times 5 \ldots$ No. $5 \times 1)$ and expressed the results in the form of a projected "heat map".

\section{Results and discussion}

\section{Discussion flow}

In Online Appendix 2, we describe the main points discussed by each group in each session, based on the members' remarks that each graphic facilitator had written down on sheets of A0-size paper. Overall, many opinions were given by individual members, but they did not seem aggregated as a group. The discussion flow of each group is summarized as follows.

As for group A, in session 1 (the current generation's perspective), participants imagined that Yahaba Town in 2050 would be a good place to live for all ages with facilities and systems in place for interaction across the ages. They proposed rebuilding houses in the order of necessity. They also proposed clarifying whom each housing unit is intended for and designing it considering people who live there. In session 2 (a future generation's perspective), they imagined that, in 2050, the development of science and technology has changed the way of living and working, and areas except for the central area around the train station and the hospital is losing population and creating disparities. They proposed consolidating and renovating existing housing and adding value to the housing with work or fields while protecting the healthy and cultured standard of living of residents. In session 3, they proposed creating complex and integrated housing with childcare provided and multiage interaction. They also proposed reviewing the organization of Yahaba town hall to cope with challenges from a holistic viewpoint. They advised the future generation not to leave problems 
Table 1 Items in the worksheet

Top: Lead/question/instructions; Bottom (in italics): Response method, choices, etc

1) concepts and viewpoints proposed in debating

a) Concepts and vision

b) Important viewpoints when deciding concepts

2) Overall principles and policies

a) Maintenance principles (multiple choice)

b) Status quo, desirable, undesirable (free form response)
Indicate your concepts (ideals) for public facilities and public housing; what should they be?

Answer freely

What viewpoints, values, and criteria did you give most importance to in deciding your concepts?

List up to five viewpoints (five items), in order of importance. Answer freely

Indicate the choice that is closest to your general view regarding the overall maintenance and management of public facilities/public housing

1) Reduce facilities, leaving only the minimum necessary facilities;

2) Increase facilities, adjusting them to suit population size;

3) Reduce facilities, adjusting them to suit population size;

4) Increase facilities, adjusting them to suit the needs of residents;

5) Reduce facilities, adjusting them to suit the needs of residents;

6) Maintain the status quo by rebuilding facilities of the same size once their usable life has expired;

7) Increase facilities because they are currently insufficient;

8) Other (give specific details)

i) What do you think will happen to the maintenance and management of public facilities/public housing if the current situation continues?

ii) What do you consider a desirable approach to the maintenance and management of public facilities/ public housing?

iii) What do you consider the most undesirable approach to the maintenance and management of public facilities/public housing?

Answer freely

Select the most essential principles when considering individual public facilities/public housing (e.g., that need high priority, or that need to be reviewed) and indicate your reasons

Select a facility name and write about your reasons and specific principles freely

4) Time scale

a) Emphasized age group when thinking of maintenance, with reasons

b) Schedule of policies and directions beyond the present
Indicate the age group that you emphasized (your target) when thinking about the maintenance of public facilities/public housing and give your reasons

Write about the age group and give your reasons freely

Indicate the maintenance and management schedule for public facilities/public housing

Construct a timeline specifying the age group and detailed policies and principles unaddressed and solve them, looking back on them. They also advised examining whether the purpose and reasons for building housing are clear.
As for group B, in session 1, participants imagined that Yahaba Town in 2050 would be convenient and safe to live in and that the number of visitors and residents is increasing. They proposed making the houses attractive in terms 
of both exterior and interior, making two wings in houses, one for the young and one for the elderly, and creating space for interaction. In session 2, they imagined that, in 2050, houses and commuters are increasing, and the transportation network is well-developed within and outside the town. Further, one information terminal available for each family connects them to the medical university for their health. They proposed letting town housing serve as a safety net and designing houses so that people who live can interact with local communities. In session 3 , they proposed building town housing from a welfare perspective for single elderly and vulnerable people and creating buildings that are easy to maintain to use for a long time safely. They also proposed making it easier for residents to produce what they need by themselves, such as solar power, to use surplus money elsewhere. They advised the future generation not to leave problems unaddressed and solve them looking back on them and to think about the future when they think about what they should do now.

As for group C, in session 1, participants imagined that Yahaba Town in 2050 would be aging and focusing on improving health. They proposed that each facility has multiple functions and incorporates shops from the private sector. In session 2, they imagined that, in 2050, the area around the medical university is convenient to live in while the other areas are more depopulated. While they maintained the policies mentioned in session 1, discussions related to the medical university have increased; for example, they proposed characterizing Yahaba Town as a town of longevity in cooperation with the medical university. In session 3 , they proposed developing facilities to promote the attractiveness of Yahaba Town concerning health, convenience, and profitability. They advised the future generation to develop facilities that make use of the town's nature, make profits, and respond to disasters.

As for group D, in session 1, participants hoped Yahaba Town in 2050 to be convenient in many ways such as shopping, transportation, medical care, and work. They suggested making existing facilities more comfortable to use in terms of location, access, information, and fees, rather than building new ones because they recognized that many facilities existed currently but were not widely used. In session 2 , they imagined that, in 2050, the population has increased, and the road network has been developed. They gave opinions for each type of public facility, safe roads, convenient town hall, some variations of town housing, and school maintenance. In session 3, they suggested allowing neighboring townspeople to use the facilities of Yahaba Town to generate interaction and revenue. They were also concerned about the difference in the development level between the east and west sides of the railroad tracks. Their advice to the future generation was optimistic that the town would be okay as before.

What changes have occurred in the attitudes of the participants as a result of the above discussions? The following sections address this question based on the participants' answers in the questionnaires and worksheets to which they responded after each session.

\section{Analysis of the questionnaires}

\section{Change in perceptions and evaluations over sessions}

There was a marginally significant main effect of session for several items in the questionnaire. Detailed results from ANOVA for the relevant items are shown in Table 2.

Changes in responses between the first and second sessions can be regarded as the effect of the switch in perspective from the current generation to the future generation. A change in responses to the item "Yahaba's welfare is a serious problem" was observed (Table 2): in the second session, welfare was not considered such a serious problem compared with the first session. For the questions "Yahaba's financial state is a serious problem," "Yahaba's population is a serious problem" and "Yahaba's welfare is a serious problem," the average values in the first session were $M$ (mean value) $=4.23$ for financial state, $M=4.00$ for population, and $M=4.62$ for welfare, which shows that, in comparative terms, welfare was seen as a serious problem. However, in the second session, the respective average values were

Table 2 Mean, standard deviation, and $F$ value for the difference between sessions

\begin{tabular}{|c|c|c|c|c|c|c|c|}
\hline & \multicolumn{2}{|c|}{ Session 1} & \multicolumn{2}{|c|}{ Session 2} & \multicolumn{2}{|c|}{ Session 3} & \multirow[t]{2}{*}{$F$ value } \\
\hline & Mean & SD & Mean & SD & Mean & SD & \\
\hline Yahaba's welfare is a serious problem & 4.62 & 0.64 & 4.39 & 0.66 & 4.56 & 0.62 & $2.56^{+}$ \\
\hline The people of today are responsible for the issues being debated & 3.65 & 1.09 & 3.25 & 0.94 & 4.00 & 0.79 & $3.62 *$ \\
\hline $\begin{array}{l}\text { I want my children's and grandchildren's generations to continue } \\
\text { living in Yahaba }\end{array}$ & 3.58 & 1.03 & 3.96 & 1.30 & 4.33 & 0.91 & $4.64^{*}$ \\
\hline Yahaba will probably be a comfortable place to live in 2050 & 3.72 & 0.79 & 4.00 & 0.85 & 4.22 & 0.73 & $3.19^{+}$ \\
\hline
\end{tabular}

SD stands for standard deviation

${ }^{*} p<0.05 .{ }^{+} p<0.10$ 
$M=4.43$ for financial state, $M=4.48$ for population, and $M=4.39$ for welfare, indicating a significant decline in the importance attached to welfare when the perspective changed to that of the future generation. In the first session, when the participants considered issues solely from the standpoint of the current generation, they assessed financial state and population as not being of particular importance in a relative sense. However, in switching perspectives in the second session, they revised their assessment of the importance of these issues for Yahaba as a town. Arguably, financial state and population are more likely to be inherited from the previous generation compared with welfare which can be somehow dealt with within a generation, and such differences seem to have been reflected upon by the results. Thinking from the viewpoint of the future generation seemed to have caused a change in the criteria that participants used to evaluate Yahaba.

In the third session, the participants were instructed to think from whichever standpoints (current generation and future generation) they preferred. Responses after the third session reflected more positive assessments than those after the first session for two items: "I want my children's and grandchildren's generations to continue living in Yahaba," and "Yahaba will probably be a comfortable place to live in 2050" as demonstrated in Table 2. The responses to these items became progressively more positive from the first session, through the second, to the third, meaning that participant assessments of the future of Yahaba seemed to steadily move in a positive direction. For example, regarding the item "Yahaba will probably be a comfortable place to live in 2050", the mean values for the first, second and third sessions were $3.72,4.00$ and 4.22, respectively (Table 2). In the second session, there appeared to be a revision in the criteria used to evaluate Yahaba as a result of thinking from the standpoint of the future generation; subsequently, in the third session, when assessing the future of Yahaba based on the adjusted criteria, the participants tended more towards envisioning Yahaba as a comfortable place to live in the future. Such a positive attitude taken through the experience of imaginary future generations is consistent with the observation of Hara et al. (2019), who fixed each group's standpoint through all the sessions.

Change was also observed between the responses from the second and third sessions. The changes between the first and second and between the first and third sessions were about the perceptions of Yahaba, but the change observed between the second and third sessions was for the item "The people of today are responsible for the issues being debated". The mean values for the item in the second and third sessions were 3.25 and 4.00 , respectively (Table 2 ). After experiencing the debates from both standpoints - the current and future generations - and then later choosing to consider the issues from either standpoint or from the standpoints of both, the participants showed a stronger awareness of the current generation's responsibility.

More than simply a change in participant perceptions of Yahaba, this was a change in their perception of the relationship between the current and future generations. In general, perceiving responsibility tends to lead to helping or pro-environmental behavior. Recognizing responsibility can lead the current generation to pass on the benefits they have enjoyed to future generations-as opposed to greedily serving only their own self-interests (Latané and Darley 1970; Hirose 1994). The experience of thinking from the standpoint of future generations, and, furthermore, thinking from the standpoints of both current and future generations, has the potential not only to benefit Yahaba, but also to lead to a broad and fresh appreciation of the relationship between the current and future generations.

\section{Viewpoint-sharing (dual perspectives)}

One of the important findings of the study relates to the notion of "viewpoint-sharing (dual perspectives)". We based this notion on the positive correlation $(r=0.52, p<0.05)$ we found between responses to "In today's debate, I thought about things from the standpoint of people living now" and "In today's debate, I thought about things from the standpoint of future generations," both of which were questionnaire items in the third session survey. This correlation suggests that the two perspectives should not be thought of as oppositional. Table 3 details how the respondents rated their level of use of each of the two standpoints. As indicated, 14 of the 20 respondents to the third session questionnaire gave the same response (i.e., the difference was 0 ) for future generation thinking and for present generation thinking. It appears after the sessions that the more people think about things from the standpoint of those living today, the more they also think about things from the standpoint of future generations. Taken together, then, these two items could be considered a measure (Cronbach's $\alpha=0.68$ ) of "thinking from the standpoints of both generations".

We rated the degree of thinking from the standpoints of both generations (i.e., the degree of viewpoint-sharing) as either low, medium, or high and examined the influence of this indicator in the third session. Participants whose responses were 3 or less (totally disagree, disagree, or neither agree nor disagree) for either of the two viewpoints were classified as members of the "low" group $(n=6)$; those with a response of 4 (agree) for both viewpoints were classified as members of the "medium" group $(n=10)$; and those with a response of 5 (very much agree) for both viewpoints or a score of 5 for one and a score of 4 for the other were classified as members of the "high" group $(n=4)$. 
Table 3 Cross-tabulation of ratings for each of the standpoints $(n=20)$

"In today's debate, I thought about things from the standpoint of future generations"

\begin{tabular}{|c|c|c|c|c|c|c|}
\hline & & $\begin{array}{l}1 \text { Totally } \\
\text { disagree }\end{array}$ & 2 Disagree & $\begin{array}{l}3 \text { Neither } \\
\text { agree nor } \\
\text { disagree }\end{array}$ & 4 Agree & $\begin{array}{l}5 \text { Very } \\
\text { much } \\
\text { agree }\end{array}$ \\
\hline \multirow{5}{*}{$\begin{array}{l}\text { "In today's debate, I thought about things from } \\
\text { the standpoint of people living now" }\end{array}$} & 1 Totally disagree & 0 & 0 & 0 & 0 & 0 \\
\hline & 2 Disagree & 0 & 0 & 1 & 0 & 0 \\
\hline & 3 Neither agree nor disagree & 0 & 0 & 2 & 0 & 0 \\
\hline & 4 Agree & 0 & 2 & 1 & 10 & 1 \\
\hline & 5 Very much agree & 0 & 0 & 0 & 1 & 2 \\
\hline
\end{tabular}

In order to further explore characteristics of those participants who exhibited "high viewpoint-sharing," we performed an one-way ANOVA (three levels) using participant responses to the third session questionnaire, followed by an application of Tukey's test.

Table 4 summarizes the third-session items for which there was a significant difference between the viewpointsharing groups, along with the statistical details. As a result, the following three items showed viewpoint to be a significant factor, with a significant difference between the low and high viewpoint-sharing groups: "The people of today are responsible for the issues being debated", "We must pass on to future generations the things we in the present are enjoying", and "The themes discussed in this debate are serious problems now".

These items pertain to the relationship between the current and future generations, suggesting that participants with a high degree of viewpoint-sharing re-thought their assessment of things in the present and recognized their importance, thereby developing a desire to pass these things on to the future generation and feeling a sense of responsibility to ensure that this is done. The relationship between the current and future generations is not oppositional in the sense of one depriving the other of resources; rather, the future generation may be thought of as a partner to which the present generation would leave an inheritance.

Further, for the items related to the conclusions of discussion "Whatever the conclusion, the future generation would probably accept the reasons for the Yahaba Plan 2050 proposal" and "The future generation would probably accept the conclusions discussed in this session," viewpoint was a significant factor, showing a difference between the low and high viewpoint-sharing groups (Table 4). In addition, for the item "The reasons for the proposal of Yahaba Plan 2050 are justifiable," a significant difference was observed between the low or medium and high viewpoint-sharing groups. These findings suggest that individuals with a high degree of viewpoint-sharing are likely capable of overviewing the relationships between the current and future generations. It can also be inferred that these participants attached greater importance to the acceptability of their conclusions to the citizens of the future generation.

Table 4 Items in the third-session questionnaire showing a significant difference between viewpoint-sharing groups (mean and standard deviation given for each viewpoint-sharing group)

\begin{tabular}{|c|c|c|c|c|c|c|c|}
\hline & \multicolumn{2}{|l|}{ Low } & \multicolumn{2}{|c|}{ Medium } & \multicolumn{2}{|l|}{ High } & \multirow[b]{2}{*}{$F$ value } \\
\hline & Mean & SD & Mean & SD & Mean & SD & \\
\hline The people of today are responsible for the issues being debated & 3.33 & 0.82 & 4.20 & 0.63 & 4.50 & 0.58 & $4.36^{*}$ \\
\hline We must pass on to future generations the things we in the present are enjoying & 3.17 & 1.17 & 3.80 & 0.42 & 4.50 & 0.58 & $3.89 *$ \\
\hline The themes discussed in this debate are serious problems now & 3.67 & 0.52 & 4.20 & 0.42 & 4.50 & 0.58 & $4.03^{*}$ \\
\hline $\begin{array}{l}\text { Whatever the conclusion, the future generation would probably accept the reasons for } \\
\text { the Yahaba Plan } 2050 \text { proposal }\end{array}$ & 3.33 & 0.52 & 3.80 & 0.79 & 4.75 & 0.50 & $5.38 *$ \\
\hline The reasons for the proposal of Yahaba Plan 2050 are justifiable & 3.00 & 0.63 & 3.70 & 0.48 & 4.75 & 0.50 & $12.88 * *$ \\
\hline The future generation would probably accept the conclusions discussed in this debate & 3.17 & 0.75 & 3.70 & 0.67 & 4.50 & 0.58 & $4.57 *$ \\
\hline Welfare is a serious problem for Yahaba & 4.00 & 0.71 & 4.67 & 0.50 & 5.00 & 0.00 & $4.58 *$ \\
\hline Those policies are feasible & 3.40 & 0.55 & 4.00 & 0.50 & 4.67 & 0.58 & $5.57 *$ \\
\hline Leaving room for future people to make their own decisions & 3.80 & 0.45 & 4.56 & 0.53 & 4.67 & 0.58 & $4.16^{*}$ \\
\hline
\end{tabular}

$* * p<0.001, * p<0.05$ 
For the item related to the evaluation of Yahaba, "Welfare is a serious problem for Yahaba," viewpoint was again a significant factor, with the high viewpoint-sharing group considering the problem to be more serious. Meanwhile, a look at the difference between the first and third sessions reveals that a change occurred following the second session, in which participants debated from the standpoint of the imaginary future generation. Specifically, participants tended to rate the welfare issue as less serious in the second and third session than in the first session (Table 2).

With regard to policy-making aspects, there was a significant difference between individuals in the low and high viewpoint-sharing groups for the item "Those policies are feasible". There was also a significant difference for the item "Leaving room for future people to make their own decisions" between the low and medium viewpoint-sharing groups, with those from the lower group attaching less importance to the item. These items address the practicability of the current generation exercising its responsibility and the desirability of giving future generations enough space to work at resolving their own problems in their own way. By thinking from the standpoints of both current and future generations, the best possible policies for the future are more likely to be pursued. From our ANOVA between the first through third sessions, no difference in individuals was observed in terms of the issues considered important when implementing policy. However, a difference was found in the items considered important between the low and high viewpoint-sharing groups.

\section{Worksheet analysis}

The results of analyzing items given most importance by the four groups (A, B, C and D) in each session are shown in the form of a heat map (Fig. 2). As can be seen, there was a clear change in the items considered important from one session to the next.

In the first session, importance was attached to the physical aspects of facilities and to improving the function of public facilities, as reflected in basic judgments concerning whether public facilities should remain, in thinking about the needs of public facility users, and in wanting to enable people to live in and use facilities safely by addressing issues of security and barrier-free functionality. With respect to public transport, impartially enabling everyone, including those from outside the town, to use these public facilities was also considered important.

In the second session, participants continued to attach importance to improving the function of public facilities; however, the focus notably shifted to quality of life issues for those using the facilities, e.g., enabling residents and users to live and use the facilities with peace of mind, and fostering a culturally satisfying lifestyle. In addition, consideration was given to the sustainability of various aspects and elements of Yahaba, and to providing easy access for facility users, e.g., by considering car access. Further, on average, greater attention was paid to the cost required for maintenance and management than other sessions. In general, participants tended to prioritize quality of life issues and to express empathy not only for the citizens of future generations, but also the people presently living in Yahaba.

In the third session, the focus widened-from public facilities and their users to the broader district and community. The discussion centered largely on the community as a whole, as evidenced by consideration of whether proposals were in accordance with the values of the town's citizens, consideration of the relationship with local residents, and ensuring that buildings and places could be used impartially by all. Accordingly, consideration turned to the concept of "outsiders" (importance was given the issue of use by people from outside the town).

We also uncovered some interesting characteristics of discussion contents by group and session. By first quantifying the intensity of topic occurrence by group and session (Table 5), and then conducting a cluster analysis of results (Fig. 3), we found that the tendency of occurrence for topics in group A in the third session (hereinafter, A-3) was
Fig. 2 Heat map showing the transition of focus on important topics by session. Darker color indicates greater focus

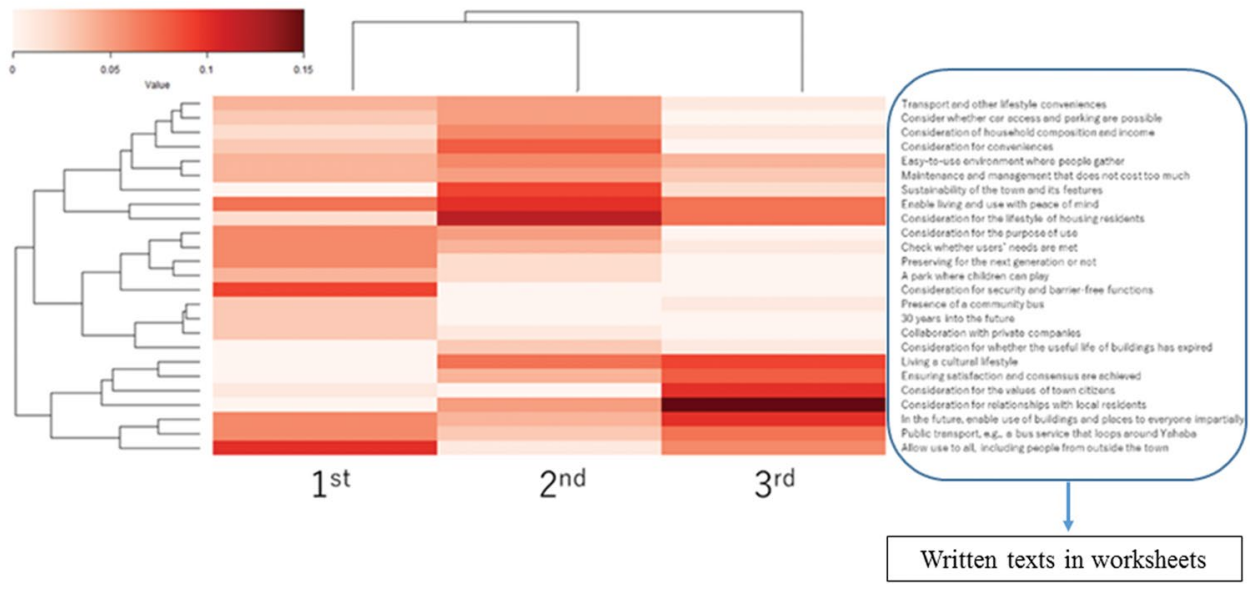


Table 5 Quantified intensity of topic occurrence by group and session

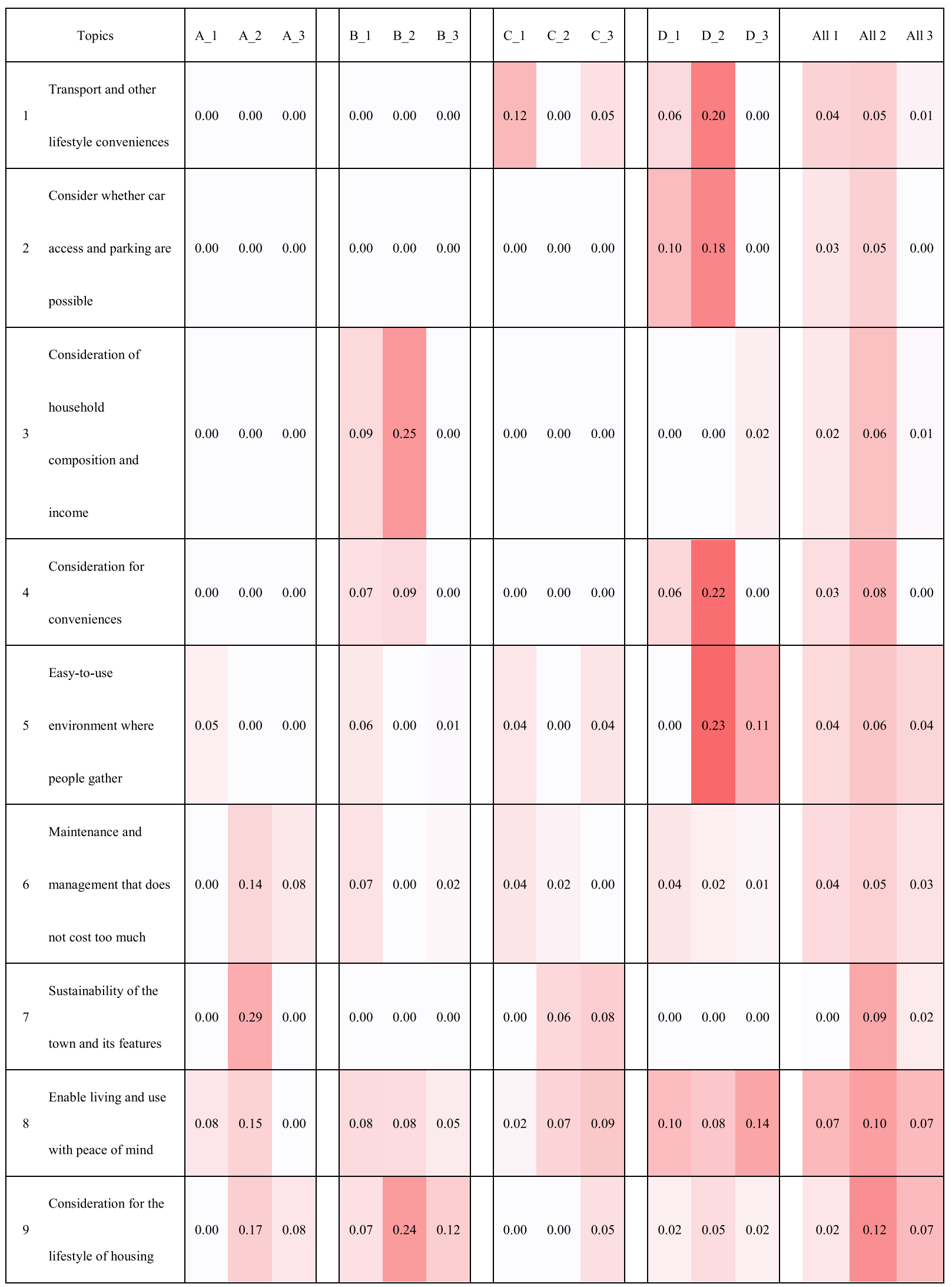


Table 5 (continued)

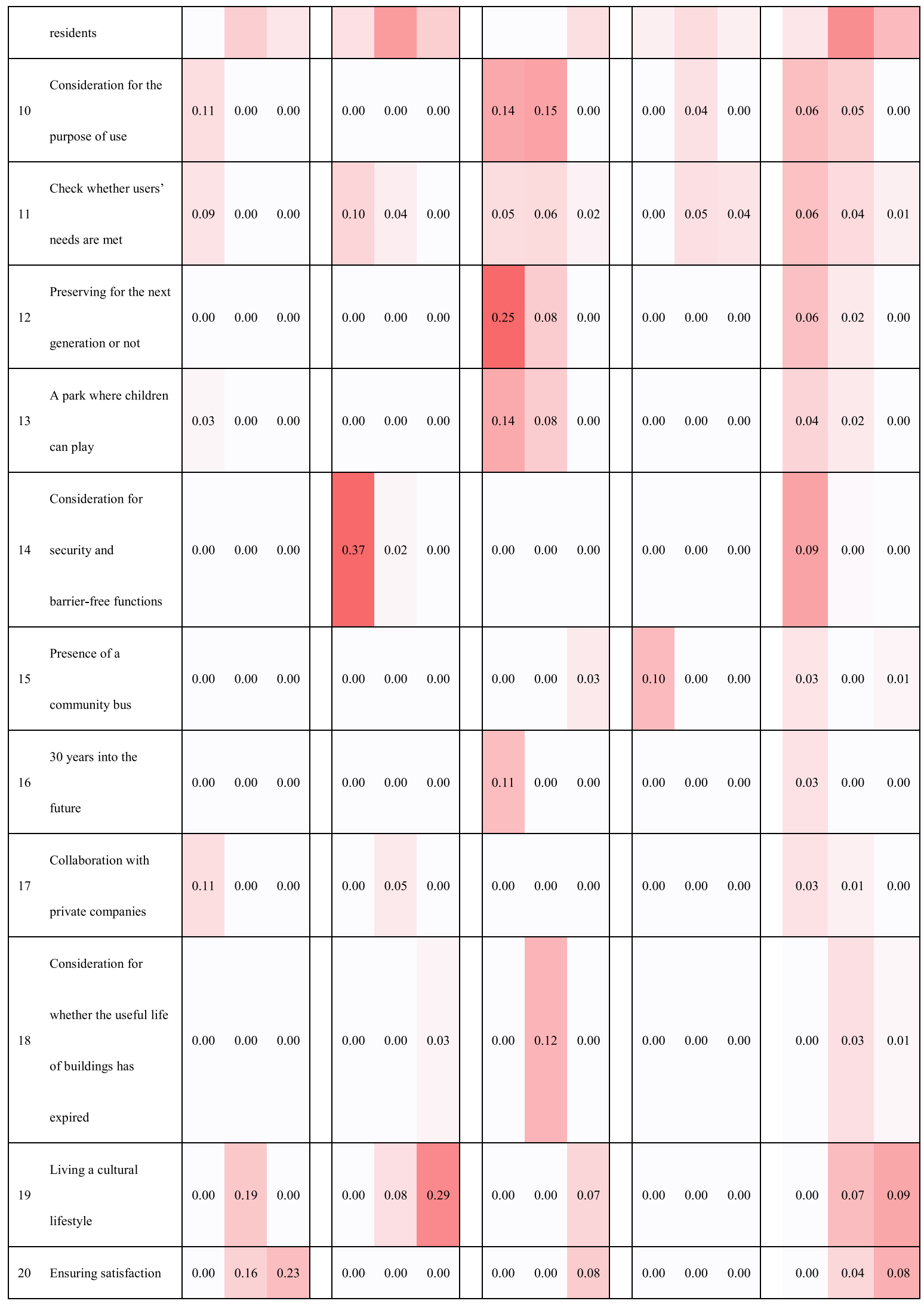


Table 5 (continued)

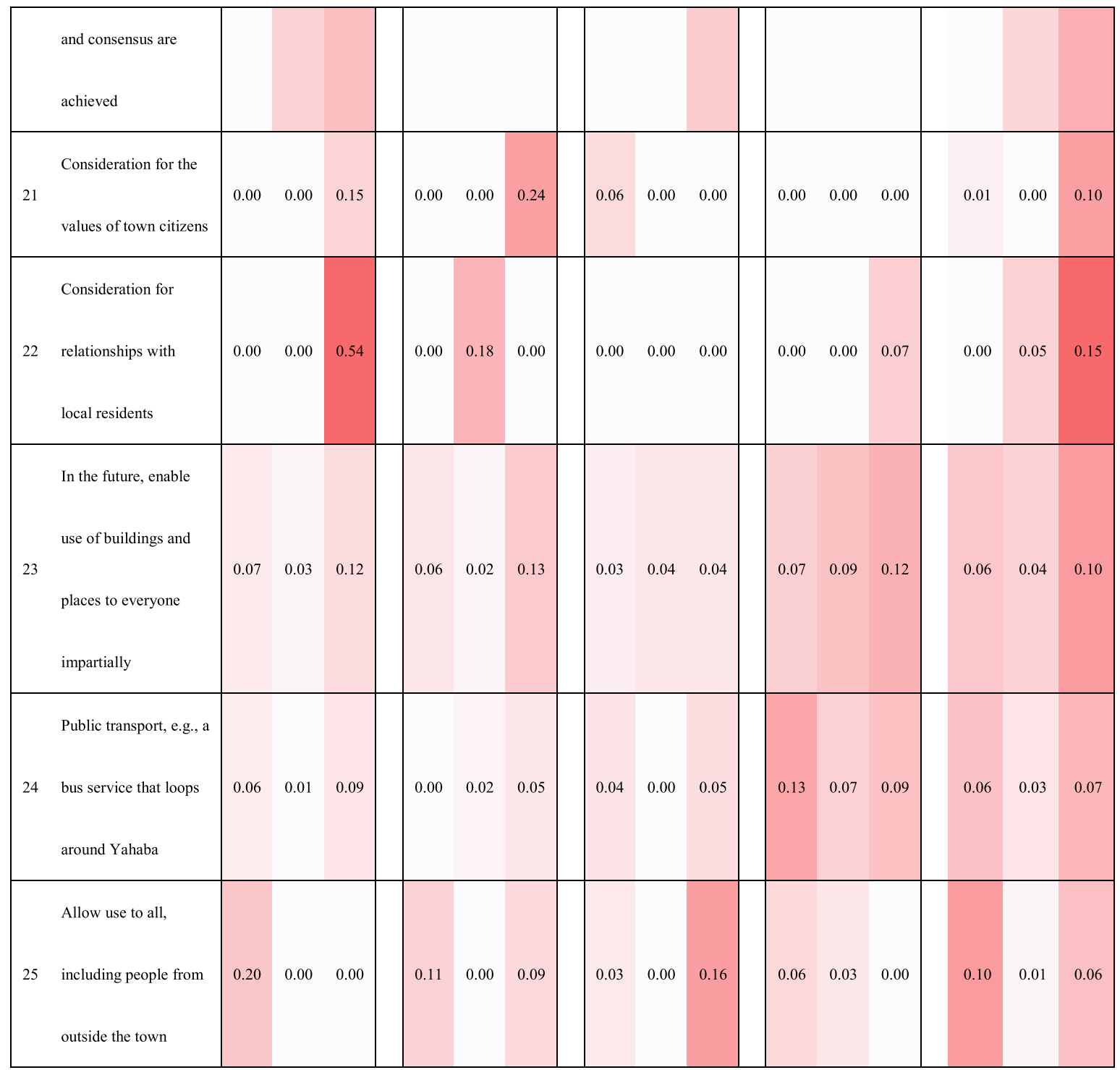

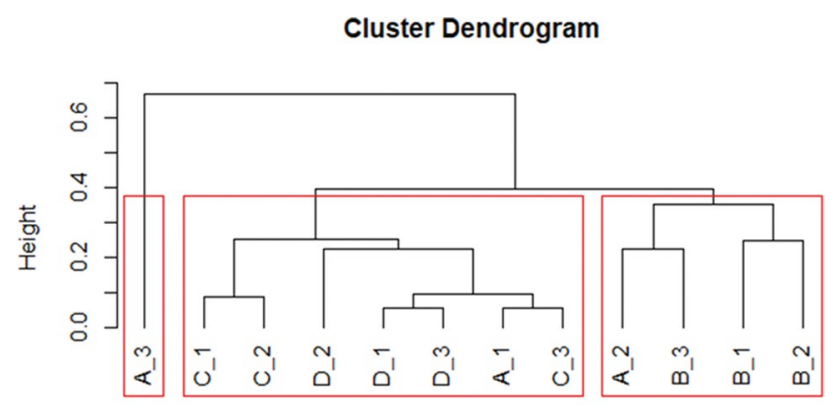

m1dist

hclust (" , "ward.D") clustered away significantly from the other groups and sessions. A-3 could be characterized as emphasizing two items: "Thinking about the relationship with local residents" and "Ensuring that satisfaction and consensus are achieved". A-2, B-1, B-2, and B-3 formed another cluster. The feature of this cluster could be interpreted as a focus on the principle that since public facilities and public housing are by nature "public," it is essential to consider users above all else (e.g., "Enabling use with peace of mind" and "Living a cultural lifestyle"). The third cluster reflected a focus on convenience, with considerations such as "Mobility and other lifestyle convenience" and "An easy-to-use environment where people gather".

Fig. 3 Cluster analysis results (cluster dendrogram) 


\section{Conclusions}

We held participatory deliberations in the Japanese town of Yahaba, where participants made decisions and provided feedback after sequentially taking on the viewpoint of the current generation and the viewpoint of an imaginary future generation. We then investigated the influences of this experience on deliberation outcomes and the decision-making process. Based on analyses of questionnaire responses and worksheet reports submitted by participants, we showed that through the experience of participating in debate and making decisions from the standpoints of both current and future generations, a self-reflective viewpoint was created and a change in perceptions and judgement clearly occurred. For instance, the town of Yahaba was assessed more positively by participants over time. Participants also developed a greater awareness of the current generation's responsibility. Further, after debating in the second session as members of the imaginary future generation, participants appeared to exhibit greater empathy, not only for future generations, but also for their neighbors in the present.

Another important finding is the development of shared viewpoints. Notably, there appeared to be no conflict between the standpoints/viewpoints of the current and future generations; the two appeared to coexist within individuals. This supports the hypothesis of Nakagawa et al. (2017) who interviewed participants acting as members of an imaginary future generation in participatory deliberations (Hara et al. 2019) that overarching perspective of both current and future generations was created within individuals. Moreover, the higher the degree of viewpoint-sharing, the more strongly the participants felt the responsibility of the current generation and the more emphatically they recognized the need to pass things on to future generations. In addition, when deciding policies, members of the high viewpoint-sharing groups attached greater importance to "feasibility" and "leaving room for future generations to make their own choices".

Study findings associated with individual self-reflective viewpoints and a change in the normative aspects of desirable visions (which are consistent with the findings of Hara et al. 2019) provide insight into how the futurability of the individual can be activated, giving a clue to how we might create institutions and social systems that facilitate sustainable decision-making by taking into account the benefits to future generations. For example, a session where members take the standpoint of an imaginary future generation after discussing as the current generation, as usual, could also be introduced into policy arena, such as local government officials' discussions and local council committees.

The approach applied in the present study, as described in Fig. 1, proved to be effective in terms of leading individuals to detach themselves from their normal self-interests and assume a self-reflexive viewpoint. Nonetheless, there would be many ways to activate futurability. Future studies should include a deeper investigation of the triggering factors and personal attributes of individuals that activate futurability. To this end, it will be necessary to accumulate detailed case studies involving the practice of participatory future design, along with large scale survey data. This will help identify appropriate social systems and institutions that support decision-making that takes into account the impact of present decisions on future generations.

Future Design aims to cope with intergenerational conflicts and to facilitate social transformation towards sustainability by incorporating the perspectives of future generations into present-day discussions. In this study, we identified some of the effects of experiencing the perspectives of an imaginary future generation. There remains a need for a more detailed study to find effective ways to implement this method in real-world policy formulation processes.

Supplementary Information The online version contains supplementary material available at https://doi.org/10.1007/s11625-021-00918-x.

Acknowledgements This study was supported by Grants-in-Aid for Scientific Research (Research Project Numbers: 16K12660 and 17H00980) from the Japan Society for the Promotion of Science. This study was also supported by the Project "Microeconomics, Macroeconomics, and Political Philosophy toward Economic Growth" undertaken at Research Institute of Economy, Trade and Industry (RIETI), and Research Institute for Humanity and Nature Project No.14200122.

Open Access This article is licensed under a Creative Commons Attribution 4.0 International License, which permits use, sharing, adaptation, distribution and reproduction in any medium or format, as long as you give appropriate credit to the original author(s) and the source, provide a link to the Creative Commons licence, and indicate if changes were made. The images or other third party material in this article are included in the article's Creative Commons licence, unless indicated otherwise in a credit line to the material. If material is not included in the article's Creative Commons licence and your intended use is not permitted by statutory regulation or exceeds the permitted use, you will need to obtain permission directly from the copyright holder. To view a copy of this licence, visit http://creativecommons.org/licenses/by/4.0/.

\section{References}

Clark WC, Dickson NM (2003) Sustainability science: the emerging research program. Proc Natl Acad Sci USA 100:8059-8061

Clauset A, Newman MEJ, Moore C (2004) Finding community structure in very large networks. Phys Rev E 70(6):066111

Eickhoff P, Geffer SG (2009) Power of imagination studio: a further development of the futureworkshop concept. In: Holman P, Devane T, Cady S (eds) The change handbook: the definitive resource on today's best methods for engaging whole systems. Berrett-Koehler Publishers, San Francisco, pp 27-35, Trade Paperback 
Fehr-Duda H, Fehr E (2016) Sustainability: game human nature. Nature 530:413-415

Fischbacher U, Gächter S, Fehr E (2001) Are people conditionally cooperative? Evidence from a public goods experiment. Econ Lett 71:397-404

Fortunato S (2010) Community detection in graphs. Phys Rep 486:75-174

Fries CC (1952) The Structure of English (Harcourt Brace)

Hara K, Saijo T (2017) Future design: evidence and insights from participatory deliberations. J Jpn Soc Water Environ 40(4):112116 ((In Japanese))

Hara K, Kuroda M, Kurimoto S, Saijo T (2019) Reconciling intergenerational conflicts with imaginary future generations - evidence from a participatory deliberation practice in a municipality in Japan. Sustain Sci 14(6):1605-1619

Hirose Y (1994) Determinants of environment-conscious behavior. Jpn J Soc Psychol 10(1):44-55 ((in Japanese))

Iacovidou E, Wehrmeyer W (2014) Making sense of the future: visions and transition pathways of laypeople and professionals from six EU countries. Glob Bioethics 25(4):211-225

IPCC (2014) Climate change 2014: mitigation of climate change. In: Edenhofer O, Pichs-Madruga R, Sokona Y, Farahani E, Kadner S, Seyboth K, Adler A, Baum I, Brunner S, Eickemeier P, Kriemann B, Savolainen J, Schlömer S, von Stechow C, Zwickel T, Minx JC (eds) Contribution of working group III to the fifth assessment report of the intergovernmental panel on climate change. Cambridge University Press, Cambridge

Kamijo Y, Komiya A, Mifune N, Saijo T (2017) Negotiating with the future: incorporating imaginary future generations into negotiations. Sustain Sci 12(3):409-420

Kates RW et al (2001) Sustainability science. Science 292(5517):641-642

Komiyama H, Takeuchi K (2006) Sustainability science: building a new discipline. Sustain Sci 1(1):1-6

Latané B, Darley JM (1970) The unresponsive bystander: why doesn't he help?, Century Psychology Series. Appleton-Century Crofts, New York

Lazarsfeld PE, Barton AH (1951) Qualitative measurement in the social sciences, classification, typologies, and indices. In: Lerner D, Lasswell HD (eds) The policy sciences: recent developments in scope and method. Stanford University Press, Stanford, pp $180-188$

Ligtvoet A, Cuppen E, Ruggero OD, Hemmes K, Pesch U, Quist J, Mehos D (2016) New future perspectives through constructive conflict: Exploring the future of gas in the Netherlands. Futures 78-79:19-33

Nakagawa Y, Saijo T (2020) Future design as a metacognitive intervention for presentism. Sustainability 12:7552

Nakagawa Y, Hara K, Saijo T (2017) Becoming sympathetic to the needs of future generations: a phenomenological study of participation in future design workshops, Kochi University of Technology, Social Design Engineering Series, SDES-2017-4

Rockström J et al (2009) A safe operating space for humanity. Nature 461(7263):472-475

Saijo T (ed) (2015) Fyūchā Dezain: Nana Sedai Saki o Misueta Shakai (Future design: a society looking ahead for seven generations), Keiso Shobo (in Japanese)

Saijo T (2017) Future design. Econ Rev 68(1):33-45 ((in Japanese))

Saijo T (2018) Future design: succeeding a sustainable nature and society to future generations. Rev Environ Econ Policy Stud 11(2):29-42 ((in Japanese))

Saijo T (2019) Future design. In: Laslier M, Sanver Z (eds) Future of economic design: the continuing development of a field as envisioned by its researchers. Springer, Berlin

Saijo T (ed) (2020a) Future design: incorporating preferences of future generations for sustainability. Springer, Berlin

Saijo T (2020b) Future design: bequeathing sustainable natural environments and sustainable societies to future generations. Sustainability 12(16):6467

Sapolsky RM (2012) Super humanity. Sci Am 307(3):40

Shahrier S, Kotani K, Saijo T (2017) Intergenerational sustainability dilemma and a potential solution: future ahead and back mechanism, Kochi University of Technology, Social Design Engineering Series, SDES-2017-9

Sharot T (2011) The optimism bias. Curr Biol 21(23):R941-R945

Sherstyuk K, Tarui N, Ravago MV, Saijo T (2016) Intergenerational games with dynamic externalities and climate change experiments. J Assoc Environ Res Econ 3(2):247-281

Suga S, Oi K, Harasawa H (1993) Study of the awareness of local residents on an expanse of water by a free association test and cluster analysis. J Jpn Soc Civ Eng No.458/IV-18:91-100 (in Japanese)

Sugino H, Hayashi N, Sekiguchi T, Terada Y (2017) Understanding the image of sea for Japanese people by free association questionnaire. J Coast Zone Stud 30(1):29-40 ((in Japanese))

Timilsina RR, Kotani K, Nakagawa Y, Saijo T (2019) Accountability as a resolution for intergenerational sustainability dilemma, Kochi University of Technology, Social Design Engineering Series, SDES-2019-2

Uwasu M, Kishita Y, Hara K, Nomaguchi Y (2020) Citizen-participatory scenario design methodology with future design approach: a case study of visioning for low-carbon society in Suita City, Japan. Sustainability 12(11):4746

Publisher's Note Springer Nature remains neutral with regard to jurisdictional claims in published maps and institutional affiliations. 